\title{
The impact of different exercise modalities on vasodilation and shear rate patterns in children.
}

\author{
Ali M McManus ${ }^{1}$, Nathan R Sletten ${ }^{2}$, Daniel J Green ${ }^{3}$ \\ Corresponding author: Dr Ali M McManus \\ Centre for Heart, Lung and Vascular Health, School of Health and Exercise Sciences, \\ University of British Columbia, Kelowna, BC V1V V17, Canada. \\ Tel: +1 250807 8192; Email: ali.mcmanus@ubc.ca
}

This work was supported by the National Sciences and Engineering Research Council Canada Discovery Grant (2015-03647) and the National Health and Medical Research Council of Australia (1080914).

The authors have no conflicts of interest to disclose.

Key words: High-intensity interval exercise, moderate-intensity exercise, steady-state, shear rate, shear patterns, superficial femoral artery

${ }^{1}$ Centre for Heart, Lung and Vascular Health, School of Health and Exercise Sciences, University of British Columbia, Kelowna, Canada. This author takes responsibility for all aspects of the reliability and freedom from bias of the data presented and their discussed interpretation.

${ }^{2}$ Centre for Heart, Lung and Vascular Health, School of Health and Exercise Sciences, University of British Columbia, Kelowna, Canada. This author takes responsibility for all aspects of the reliability and freedom from bias of the data presented and their discussed interpretation.

${ }^{3}$ School of Human Sciences (Sport and Exercise Science), Faculty of Science, The University of Western Australia, Crawley, WA, Australia. This author takes responsibility for all aspects of the reliability and freedom from bias of the data presented and their discussed interpretation. 


\section{ABSTRACT}

Background. Exercise training can improve vascular function through anti-atherogenic effects on the vascular endothelium, a response that can be discerned following a single bout of exercise. Although characterized in adults and adolescents, the effect of exercise intensity on vasodilator function is poorly understood in children. We compared the effect of a single bout of highintensity interval exercise (HIIE) and a single bout of moderate-intensity steady state exercise (MISS) on post-exercise vasodilation and shear patterns in nine 7 to 12 year olds.

Methods. Superficial femoral artery (SFA) diameter, shear rates and flow-mediated dilation (FMD) were measured pre, immediately following (post), and 60-minutes following (post60) HIIE (six 1-minute sprints at 90\% peak power ( $\mathrm{W}_{\max }$ ), with 1-minute recovery) and MISS (15 minutes at $44 \% \mathrm{~W}_{\max }$, external work-matched to HIIE).

Results. Baseline SFA diameter increased similarly following both HIIE (pre $4.25 \pm 0.42 \mathrm{~mm}$, post $4.76 \pm 0.42 \mathrm{~mm}$ ) and MISS (pre $4.29 \pm 0.49 \mathrm{~mm}$, post $4.61 \pm 0.67 \mathrm{~mm}$ ), returning to preexercise values post60. Blood flow and antegrade shear rate were increased following HIIE and MISS, but to a greater extent after HIIE $(P<0.05)$. Retrograde shear rate was attenuated following both exercise conditions, remaining lower post60 ( $P$ 's $<0.001)$. There was a decline in FMD post exercise (HIIE $\Delta-2.8 \%$; MISS $\Delta-2.5 \%$ ), which was no longer apparent when corrected for baseline diameter.

Conclusions. Acute bouts of external work-matched HIIE or MISS exert a similar impact on shear-mediated conduit artery vasodilation and FMD in children and this is reversed 60-minutes post-exercise. 


\section{INTRODUCTION}

It is now recognized that exercise provides a direct anti-atherogenic effect on vascular health (22). Early deterioration in vascular function, particularly alterations in endothelial integrity, are significant in the development of cardiovascular disease (1). While there is evidence of improved vascular endothelial function with exercise training in adolescents (40), little is known about the optimal exercise for vascular endothelial benefit in the child.

The response of the vascular endothelium to acute bouts of exercise provides insight into early endothelial adaptations noted with exercise training (15). The rise in blood flow to the active muscle at the onset of exercise increases shear rates, causing endothelium-dependent vasodilation (36). At least in adults and adolescents, this response appears to be intensity-dependent, with greater elevations in shear rate (10) and optimized shear rate patterns in the exercised limb (3) following higher intensity exercise. A greater vasodilatory response to high-intensity exercise has also been noted in adults $(3,9)$, although in adolescents the increase in baseline diameter was similar following moderate- and high-intensity exercise (10). In both adults and adolescents, a biphasic intensity dependent response in flow-mediated dilation (FMD) exists, with decreases in FMD immediately after high- but not moderate-intensity exercise, then subsequent upregulation, or normalization of FMD one or more hours after the cessation of high-intensity exercise $(3,4,9)$.

Although the mechanisms underlying the temporal response in FMD following high-intensity exercise remain unclear, there is some evidence in adults that sympathetic vasoconstriction may compete with endothelium dependent vasodilation causing post-exercise reductions in FMD (4). Equally, the intense pulsatile shear stimulus induced by higher intensity exercise may result in increased oxidative stress, leading to an immediate reduction in NO bioavailability (20), and hence 
a greater immediate decline in FMD. The increases in FMD in the late phase of recovery from high-intensity exercise may result from endothelial NO synthase (eNOS) upregulation (13), and this is believed to be an important transitory response that mediates longer-term FMD augmentation (28).

Endothelial adaptation to exercise of differing intensities in children is less clear. Work documenting the vasodilator and FMD responses to acute bouts of active video game play of differing intensities has shown a decrease in brachial artery FMD immediately following high-, but not low-intensity play in 10-year-olds (25). This was not attributable to differences in baseline diameter which remained constant across the conditions (25). In contrast, an increase in brachial artery FMD following supra-maximal exercise has been demonstrated (11). Importantly, the exercise bouts in both of these studies were not matched for total external work, which is critical given that the volume of exercise also influences FMD (24). It is also worth noting that both studies examined the impact of acute high intensity exercise in the non-exercising upper limb. There are known regional differences in vascular responses to exercise (21), alongside evidence that vascular disease risk is often detectable in the blood vessels of the legs rather than the arms [i.e. intermittent claudication, peripheral arterial disease] (27). Furthermore, the timecourse of the post-exercise vasodilatory response was not explored, so whether a transient biphasic response is also evident in the lower limb in children following high-intensity exercise is unknown.

The purpose of this study was to compare the time-course of vasodilator responses of the superficial femoral artery (SFA) following acute bouts of external work-matched high-intensity interval exercise (HIIE) and moderate-intensity steady-state exercise (MISS) in children. Our 
primary hypotheses were (i) baseline conduit artery diameter will be increased immediately following both HIIE and MISS, returning to pre-exercise values 60-minutes post-exercise, (ii) HIIE will result in a decreased artery function (FMD) immediately post exercise, with a subsequent increase above baseline values 60-minutes after cessation of exercise, whereas FMD will be unaltered at all time-points following MISS, (iii) antegrade shear rate will be higher and retrograde shear rate lower post HIIE, compared to MISS, and (iv) blood flow and hyperaemic shear responses will be higher post HIIE compared to MISS and will return to baseline values 60-minutes after exercise in MISS but not HIIE.

\section{METHODS}

\section{Study Participants}

Twelve children (age 7-12 years; 7 girls) were recruited, none of whom were currently training (exercise $>10$ hours per week), obese, or had a physical limitations or health condition that would impact vascular function or the ability to exercise (determined via a health history questionnaire completed by parents). Pubertal status was determined from parental-assessment of Tanner stage (29). Prior to the experimental trials the children attended the laboratory for familiarization with the equipment and procedures to be used in the study.

\section{Ethical Approval}

This study was approved by the Clinical Research Ethics Board at University of British Columbia (H16-00077). Written participant assent and parental consent were obtained prior to participation in the study. All experimental protocols and procedures conformed to the 
standards set by the Canadian Government Tri-Council Policy Statement for Integrity in Research, as well as the Declaration of Helsinki.

\section{Procedures and Experimental Design}

The children visited the laboratory three times. During the first visit, they completed anthropometric measurements and a maximal exercise test to determine their maximum Watts $\left(\mathrm{W}_{\max }\right)$. They returned to the laboratory at the same time of day on two further occasions to complete the HIIE and MISS trials. The children were asked to abstain from vigorous exercise and caffeine for 24 hours prior to each visit and not to eat at least 2 hours before the visits. Parents were asked to provide identical food on testing days and avoid foods containing fat and vitamin $\mathrm{C}$, which may acutely effect endothelial function $(31,38)$.

We used a crossover trial with two experimental conditions: HIIE and MISS. Following an unloaded 3-minute warm-up, HIIE consisted of six 1-minute sprint intervals at $90 \% \mathrm{~W}_{\max }$, each separated by 1 -minute of active recovery pedalling at $20 \% \mathrm{~W}_{\max }$. Following the same warmup, MISS entailed 15 minutes of cycling at a cadence of $70-90 \mathrm{rpm}$ at $44 \% \mathrm{~W}_{\max }$. The two trials were matched for total external work, which was calculated using the square wave method. In brief, this entails summing the total external work for the HIIE exercise and rest intervals $(90 \%, 20 \%, 90 \%, 20 \%$ etc.), then dividing the 15 -minute exercise time for MISS by the total HIIE external work to give the MISS intensity ( $\left.44 \% \mathrm{~W}_{\max }\right)$ needed to achieve the same total external work (796 \pm 163 arbitrary units). The order of completion was randomized and the conditions were completed on two separate days, separated by at least 48 hours of recovery.

Figure 1 provides a schematic of the study design and measurements. During the two experimental trials participants completed pre-exercise BP, HR and FMD assessment in the 
semi-supine position followed by HIIE or MISS. During exercise HR was assessed continuously, and in the final 15 seconds of both exercise trials the children were asked to rate their perceived exertion using a child-specific scale. HR and FMD were assessed post $(<10$ minutes) and post60 in the semi-supine position.

$<$ Insert figure 1 about here>

\section{Laboratory Measures}

Stature and body mass were measured barefoot in light clothing ( $\mathrm{t}$-shirt and shorts) to the nearest $0.1 \mathrm{~cm}$ and $0.1 \mathrm{~kg}$ using a stadiometer (Seca 217) and electronic scale (Tanita TBF410), respectively. Body mass index (BMI) was calculated as $\mathrm{kg} \cdot \mathrm{m}^{2}$ and weight status was calculated using age- and sex-specific cut-offs (12).

Baseline systolic (SBP) and diastolic blood pressure (DBP) were measured twice using a manual sphygmomanometer (Prestige Medical 79-BLK standard aneroid, Northridge CA) to the nearest $\mathrm{mmHg}$ on the right arm. Heart rate (HR) was measured continuously from pre to post60 using HR telemetry (Polar Vantage NV, Polar Electro Oy).

Participants performed a ramp maximal exercise test with supramaximal verification to determine maximal oxygen uptake $\left(\dot{\mathrm{V}}_{2 \max }\right)$ and $\mathrm{W}_{\text {max }}$. The test began with 3-minutes of unloaded pedalling followed by a workload increase depending on the stature of the child $\left(<110 \mathrm{~cm} 5 \mathrm{~W} \cdot \mathrm{min}^{-1}, 110-125 \mathrm{~cm} 10 \mathrm{~W} \cdot \mathrm{min}^{-1}, 125-150 \mathrm{~cm} 15 \mathrm{~W} \cdot \mathrm{min}^{-1},>150 \mathrm{~cm} 20 \mathrm{~W} \cdot \mathrm{min}^{-1}\right)$. A pedal cadence between 70-80 rpm was maintained throughout the test and continued until volitional exhaustion, which was defined as a drop in cadence below $60 \mathrm{rpm}$ for 5 consecutive seconds. At the end of the test the children pedalled unloaded for 5-minutes, then rested for 
10-minutes before the supramaximal verification of maximum. The supramaximal test began with a 2-minute warm-up followed by a 'step' transition to $105 \%$ of the $\mathrm{W}_{\max }$ achieved during the ramp test. This intensity was maintained until exhaustion at which point a 3-minute unloaded cool-down was completed.

Respiratory gases and ventilation were assessed breath-by-breath data using an online gas analyzer (Oxycon Pro, Carefusion). Data were subsequently time-aligned to second-by-second and processed using 15 -second bins. $\dot{\mathrm{VO}}_{2 \max }$ was defined as less than $5 \%$ difference in the slope of increase in $\dot{\mathrm{V}}_{2}$ between the maximal and supramaximal tests (7).

SFA blood flow and diameter were assessed via high-resolution Doppler with B-mode images with a $15 \mathrm{MHz}$ Broadband Multi Frequency Linear Array Transducer (Terason USMART3300 ${ }^{\mathrm{TM}}$; Teratech, Burlington, MA, USA). Following 15-minutes of supine rest a blood pressure cuff controlled with a fast deflating aneroid (Hokanson SC5, Bellevue, WA) was placed $\sim 5 \mathrm{~cm}$ above the knee, distal to the ultrasound probe. Once optimal imaging of the lumen-arterial wall interface was obtained, baseline SFA diameter, blood flow, and shear rates (mean, antegrade, retrograde) were continuously measured for 1-minute prior to cuff inflation. The cuff was inflated to $50 \mathrm{mmHg}$ above resting systolic blood pressure for 5-minutes, with measurement resuming 30-seconds prior to cuff deflation and continuing for 5-minutes post cuff deflation. Probe placement was measured from the medial epicondyle of the femur to ensure similar measurements across experimental conditions.

Semi-automated edge-detection and wall-tracking software, which is largely independent of investigator bias was used. This software provides continuous and simultaneous measurement of diameter and velocity, blood flow (lumen cross-sectional area and Doppler velocity, 
$\left[4^{*}\right.$ velocity $\left.\mathrm{cm} . \mathrm{s}^{-1}\right] /$ diameter $\mathrm{cm}$ ) and shear rate; as well as post hoc calculation of FMD. Velocity and flow were calculated from the Doppler envelope. Antegrade and retrograde blood flow and shear rates were calculated from antegrade and retrograde area under the curve data that were subsequently averaged from positive or negative data points respectively (34). Oscillatory shear index (OSI) was calculated as an indicator of the magnitude of shear oscillation, which is associated with endothelial dysfunction in adults (23).

$$
\text { OSI = |retrograde shear } \mid /(\text { antegrade shear }|+| \text { retrograde shear } \mid)
$$

FMD was not corrected for $\mathrm{SR}_{\mathrm{AUC}}$, in agreement with previous recommendations (34), but was adjusted for baseline diameter using a log-linear approach (5). A single researcher conducted all FMD measurements and analysis and was blinded to the study codes and participant files. The between-trial coefficients of variation for FMD (\%) and baseline diameter were $8.2 \%$ and $3.0 \%$, respectively.

An Eston-Parfitt curvilinear RPE scale (17) adapted for children was used to compare the perceived difficulty of the exercise trials. The RPE scale was placed in front of the children during the final 15 seconds of each exercise trial and the child was asked to look at the pictorial scale of a child cycling at different points up a slope and choose the picture that best represented how they currently felt. The scale ranged from 0 'very, very easy' to 10 'so hard I am going to stop'.

\section{Statistical Analyses}

All data are presented as means \pm SD. Within (pre, post, post60) and between (HIIE, MISS) condition main effects for baseline diameter, flow, shear rates and uncorrected SFA FMD (\%) 
were examined using repeated measures analyses of variance (RM ANOVA). Where necessary, main effects were deconstructed using $t$-tests with Bonferroni correction. A log-linear mixed model, with baseline diameter as the time-varying covariate, was used to examine the effect of exercise intensity (HIIE, MISS) on FMD (pre, post, post60). Mean differences were backtransformed to the original units of FMD (\%), providing corrected SFA FMD (\%). Mean differences in HR and RPE between the HIIE and MISS trials were assessed using independent samples $t$-test. Statistical significance was set at $P \leq 0.05$. Statistical analyses were performed using SPSS 22.0 (SPSS, Chicago, IL, USA).

\section{RESULTS Present $95 \%$ confidence intervals with $\mathrm{p}$ values.}

Three participants were excluded from the final analysis. Two did not present to each visit in the same prandial state, and one child did not achieve $\dot{\mathrm{V}}_{2 \max }$. Of the remaining children $(\mathrm{n}=9)$. six were girls. The mean age was $10.5 \pm 1.5$ years (age range 7.2 to 11.8 years). Seven were prepubertal (Tanner stage 1) and 2 girls were early-pubertal (Tanner stage 2). Mean BMI was 17.1 SD $2.3 \mathrm{~kg} \cdot \mathrm{m}^{2}$ (range 13.8 to $21.9 \mathrm{~kg} \cdot \mathrm{m}^{2}$ ), with one participant classifying as overweight. Mean body mass and stature were $36.1 \pm 8.2 \mathrm{~kg}$ and $144.3 \mathrm{SD} 10.2 \mathrm{~cm}$ respectively. Resting HR was 85 \pm 8 beats $\cdot \mathrm{min}^{-1}$ and resting SBP and DBP were $105 \mathrm{SD} 8 \mathrm{mmHg}$ and $73 \pm 8 \mathrm{mmHg}$ respectively. $\dot{\mathrm{V}}_{2 \max }$ values were as expected for the age group $\left(1.65 \mathrm{SD} 0.22 \mathrm{~L} \cdot \mathrm{min}^{-1}\right.$, range 1.40 to $2.10 \mathrm{~L} \cdot \mathrm{min}^{-}$ $\left.{ }^{1}\right)$. 
Physiological responses and perceived exertion during and after the two exercise conditions are presented in Table $1 . H R$, expressed as a percentage of maximum $\left(H R_{\max }\right)$, was significantly higher during each exercise interval of HIIE compared to the corresponding MISS stage $(t(16)=9.725$, $P<0.001$ ), with a mean $\% \mathrm{HR}_{\max }$ of $82 \mathrm{SD} 4$ and $69 \mathrm{SD} 4$ in the HIIE exercise intervals and corresponding MISS stages respectively. HR immediately post-exercise was elevated in both HIIE and MISS, but was higher following HIIE $(t(16)=2.446, P<0.05)$. HR returned to baseline 60 minutes after exercise in both trials. RPE was greater in HIIE compared to MISS post exercise $(\mathrm{t}(16)=4.131, P<0.01)$.

<Insert Table 1 about here>

There were no significant differences in baseline SFA diameter or FMD (\%) prior to the HIIE or MISS conditions between the children starting with the HIIE condition or those starting with the MISS condition, verifying that the washout period between trials was sufficient to eliminate any possible carry-over effects.

SFA diameters at baseline and with reactive hyperaemia prior to and following the HIIE and MISS trials are presented in Table 2. Baseline diameter increased to a similar extent following both HIIE and MISS $\left(\mathrm{F}(1.318,21.089)=27.108, P<0.001 ; \eta^{2}=0.629\right)$, returning to pre-exercise values 60 minutes following exercise. Delta change in baseline diameter from pre to post and post60 are presented in Figure 2, panel a and b respectively.

$<$ Insert Figure 2 about here>

Baseline SFA blood flow increased following both HIIE and MISS $(\mathrm{F}(1.172,18.749)=89.172, P$ $\left.<0.001 ; \eta^{2}=0.848\right)$, but to a greater extent following $\operatorname{HIIE}\left(\mathrm{F}(1.172,18.749)=5.697, P<0.05 ; \eta^{2}\right.$ 
$=0.263$ ). Flow remained elevated 60-minutes after both HIIE and MISS (see Table 2). Under hyperemic conditions SFA blood flow increased post HIIE and MISS $(\mathrm{F}(1.282,20.510)=46.695$, $P<0.001 ; \eta^{2}=0.745$ ), remaining elevated 60 -minutes after the two exercise conditions (see Table 2).

\section{<Insert Table 2 about here>}

SFA FMD $(\%)$ decreased following both HIIE and MISS $\left(\mathrm{F}(2,32)=8.145, P<0.01 ; \eta^{2}=0.337\right)$, returning to baseline values 60-minutes post-exercise (see Table 2). Delta change in FMD (\%) from pre to post and post60 are presented in Figure 2, panels c and d respectively. When FMD (\%) was corrected for changes in baseline diameter, there was no longer a decrease post-exercise $(P=$ 0.34, see Table 2).

Baseline mean, antegrade, and retrograde shear rates are presented in Figure 3. Mean shear rate increased following both HIIE and MISS $\left(\mathrm{F}(2,32)=190.101, P<0.001 ; \eta^{2}=0.922\right)$, but to a greater extent after HIIE $\left(\mathrm{F}(2,32)=7.941, P<0.01 ; \eta^{2}=0.332\right.$; see figure $\left.3 \mathrm{a}\right)$. Likewise, antegrade shear rate increased following HIIE and MISS $\left(\mathrm{F}(2,32)=151.601, P<0.001 ; \eta^{2}=0.905\right)$, with a greater increase after $\operatorname{HIIE}\left(\mathrm{F}(2,32)=7.310, P<0.01 ; \eta^{2}=0.314\right.$; see figure $\left.3 b\right)$. Mean and antegrade shear rates remained augmented 60-minutes post HIIE and MISS compared to preexercise values. Retrograde shear was attenuated to a similar extent following both HIIE and MISS $\left(\mathrm{F}(1.319,21.109)=35.998, P<0.001 ; \eta^{2}=0.692\right)$ and remained so 60 -minutes post-exercise (see Figure 3c).

<Insert Figure 3 about here> 
Hyperemic $\mathrm{SR}_{\mathrm{AUC}}$ was elevated following both HIIE and MISS $(\mathrm{F}(1.410,22.568)=30.122, P<$ $0.001 ; \eta^{2}=0.653$, remaining elevated above pre-exercise values 60 -minutes post-exercise in both conditions (see Table 2).

Oscillatory shear rate was reduced post exercise $\left(\mathrm{F}(1.253,20.040)=41.688, P<0.001 ; \eta^{2}=0.723\right)$, remaining lower than the pre-exercise values for both HIIE and MISS 60-minutes post-exercise (see Table 2).

\section{DISCUSSION}

These findings demonstrate that blood flow and antegrade shear rate were increased following both HIIE and MISS, with a concomitant increase in SFA diameter. Interestingly, the increases in flow and antegrade shear rate were greater following HIIE and while the increase in dilation of the SFA was similar following both exercise conditions, this was reversed 60-minutes after exercise, with blood flow and shear rates remaining elevated an hour after exercise. Contrary to our hypothesis, SFA FMD (\%) decreased after both HIIE and MISS, returning to baseline values within one hour. This post exercise reduction was negligible after correcting FMD for the increase in SFA baseline diameter.

The post-exercise SFA vasodilation noted in this study is likely mediated by the increase in antegrade shear rate following the increase in blood flow in the exercising limb (21). The changes in shear patterns we note are typical of the exercised limb (3) and would, at least in adults, be expected to augment FMD 60-minutes following exercise at a higher intensity, with no increases in retrograde shear leading to an improved oscillatory shear pattern and upregulation of eNOS (13, 21). The lack of an augmented FMD, despite optimised oscillatory shear patterns an hour after 
exercise corroborates previous findings that factors regulating FMD may differ in children compared to adults (33).

This study was intended to extend earlier findings in children $(11,25)$ by observing the temporal vasodilatory and FMD response of a lower limb vessel, as well as appropriately scaling FMD for baseline diameter at the various time-points. Uncorrected FMD can lead to erroneous conclusions, given that baseline diameter has an inverse relationship with FMD (32). We demonstrate that the post-exercise nadir in FMD observed after HIIE and MISS was negligible once FMD was corrected for baseline diameter. In contrast, post-exercise attenuation in FMD noted in adolescents and adults remained even when FMD was adjusted for increases in baseline diameter $(10,14)$. It is worth noting methodological differences between our study and prior studies. We assessed endothelial function in the exercising limb, while the adult and adolescent studies report adaptations in the non-exercised upper limb vessel and the impacts on baseline diameter may not be as large. Vessels in the non-exercised limb experience an increase in retrograde shear (21), which can increase production of reactive oxygen species (ROS) and decrease eNOS, causing a decrease in NO bioavailability (41). In contrast, we show cycling exercise, irrespective of intensity, caused a reduction in retrograde shear in the superficial femoral artery.

Exercise-induced oxidative stress has been shown to be attenuated in children compared to adults due to a blunted cytokine and immune response (35). Moreover, considering the negative relationship between exercise hypertension and FMD (19), the lower hypertensive response during exercise noted in children (39) could diminish any strain-mediated ROS generation. Exercise also produces large increases in sympathetic nerve activity (SNA) in the exercising muscle (16), which impairs vasodilatory function in adults due to competition between SNA-mediated vasoconstriction and endothelium-dependent vasodilation. Pre-pubertal children possess a greater 
proportion of type I muscle fibres compared to adolescents and adults (26) and the density of vascular $\alpha$-adrenergic receptors is believed to be lower, resulting in a smaller adrenergic response to exercise compared to adults (30). These characteristics lead to a smaller response to norepinephrine, lesser adrenergic vasoconstrictor tone, and greater vasodilatory influence compared to glycolytic type II fibres (8). Furthermore, the larger contribution of oxidative phosphorylation during exercise of higher intensities in children attenuates peripheral metabolic perturbations (6), which can impact upon plasma anti- and pro-oxidant status (18), leading to lower muscle lactate production, a blunted catecholamine response and lower efferent sympathetic excitation $(2,37)$. This may explain the maintenance of SFA blood flow 60-minutes following exercise due to a lack of vascular constrictive tone, leading to a reduction in peripheral vascular resistance through the maintenance of vasodilation in the distal arterioles.

Another possibility for the initial decline in uncorrected FMD is the cardiovascular load used in this study. Exercise with a lower cardiovascular load $\left(<100\right.$ beats $\left.\cdot \mathrm{min}^{-1}\right)$ is associated with minimal effect on FMD $(3,9)$, regardless of the vessel imaged. Reductions in FMD in healthy individuals are typically reported following exercise at intensities at or above $70 \% \mathrm{HR}_{\max }(3,9)$. Our two exercise intensities elicited 82 and $69 \% \mathrm{HR}_{\max }$ for HIIE and MISS respectively, so it is possible that the initial decrement in uncorrected FMD could be attributed to these cardiovascular loads.

\section{Study Limitations Considerations}

The strengths of the current study include utilizing total external work-matched exercise trials, assessing the time-course of post-exercise vasodilation and shear rate, and appropriate scaling for changes in baseline diameter across the multiple time points. This research is also strengthened by utilizing semi-automated edge-detection and wall-tracking software to determine FMD and 
hemodynamic responses, and rigorously following international guidelines for measurement (34). Nonetheless, some limitations exist. Unfortunately, we were unable to measure SFA diameter and shear patterns during exercise due to the technical difficulty of obtaining these while cycling. Furthermore, we did not assess endothelial-independent vasodilation via nitroglycerine, therefore our results reflect global vascular change and cannot be ascribed definitively to the endothelium or smooth muscle cell lines. We did not take measures in the fasted state as typically done in adults; however, we did track consistency of foods across the trials and the avoidance of foods that could influence vascular function. Last, with the loss of three participants from the final data analyses, the study is likely under-powered; however, this is unlikely to change the main findings.

To conclude, total external work-matched exercise, irrespective of intensity, exerts a similar impact on blood flow and shear rate patterns in children after 60-minutes of rest. The lack of an increase in FMD 60-minutes after the cessation of HIIE suggests the mechanisms which govern the FMD response to acute exercise are likely developmentally divergent.

\section{ACKNOWLEDGEMENTS}

We would like to thank Ryan Simair and Kevin Castilloux for assistance in data collection and the children who participated in this research project. 


\section{REFERENCES}

1. Aggoun Y, Szezepanski I \& Bonnet D (2005). Noninvasive assessment of arterial stiffness and risk of atherosclerotic events in children. Pediatr Res 58, 173-178.

2. Amano T, Kai S, Nakajima M, et al. Sweating responses to isometric hand-grip exercise and forearm muscle metaboreflex in prepubertal children and elderly. Exp Physiol. 2017;102(2):214-27.

3. Atkinson CL, Carter HH, Dawson EA, Naylor LH, Thijssen DH, Green DJ. Impact of handgrip exercise intensity on brachial artery flow-mediated dilation. Eur J Appl Physiol. 2015a;115(8):1705-13.

4. Atkinson CL, Lewis NCS, Carter HH, Thijssen DHJ, Ainslie PN, Green DJ. Impact of sympathetic nervous system activity on post-exercise flow-mediated dilatation in humans. J Physiol. 2015;593(23):5145-56.

5. Atkinson G, Batterham AM. Allometric scaling of diameter change in the original flowmediated dilation protocol. Atherosclerosis. 2013;226(2):425-7.

6. Barker AR, Welsman JR, Fulford F, Welford D, Armstrong N. Quadriceps muscle energetics during incremental exercise in children and adults. Med Sci Sports Exerc. 2010;42(7):1303-13.

7. Barker AR, Williams CA, Armstrong N. Establishing maximal oxygen uptake in young people during a ramp cycle test to exhaustion. Br J Sports Med. 2011;45(6):498-503.

8. Behnke BJ, Armstrong RB, Delp MD. Adrenergic control of vascular resistance varies in muscles composed of different fiber types: influence of the vascular endothelium. Am J Physiol Regul Integr Comp Physiol. 2011;301(3):R783-90. 
9. Birk GK, Dawson EA, Batterham AM, et al. Effects of exercise intensity on flow mediated dilation in healthy humans. Int J Sports Med. 2013;34(5):409-14.

10. Bond B, Hind S, Williams CA, Barker AR. The acute effect of exercise intensity on vascular function in adolescents. Med Sci Sports Exerc. 2015;47(12):2628-35.

11. Chuensiri N, Tanaka H, Suksom D. The acute effects of supramaximal high-intensity intermittent exercise on vascular function in lean vs. obese prepubescent boys. Pediatr Exerc Sci. 2015;27(4):503-9.

12. Cole TJ, Bellizzi MC, Flegal KM, Dietz WH. Establishing a standard definition for child overweight and obesity worldwide: international survey. BMJ. 2000;320(7244):1240-3.

13. Davies PF, Spaan JA, Krams R. Shear stress biology of the endothelium. Ann Biomed Eng. 2005;33(12):1714-8.

14. Dawson EA, Green DJ, Cable NT, Thijssen DH. Effects of acute exercise on flow-mediated dilatation in healthy humans. J Appl Physiol. 2013;115(11):1589-98.

15. Dawson EA, Cable NT, Green DJ, Thijssen DGJ. Do acute effects of exercise on vascular function predict adaptation to training? Eur J Appl Physiol. 2018;118, 523-530

16. DiCarlo SE, Chen CY, Collins HL Onset of exercise increases lumbar sympathetic nerve activity in rats. Med Sci Sports Exerc. 1996;28(6):677-84.

17. Eston RG, Lambrick DM, Rowlands AV. The perceptual response to exercise of progressively increasing intensity in children aged 7-8 years: validation of a pictorial curvilinear ratings of perceived exertion scale. Psychophysiol. 2009;46(4):843-51.

18. Ghiselli A, Serafini M, Natella F, Scaccini C. Total antioxidant capacity as a tool to assess redox status: critical view and experimental data. Free Radic Biol Med. 2000;29(11):110614. 
19. Gonzales JU, Thompson BC, Thistlethwaite JR, Scheuermann BW. Association between exercise hemodynamics and changes in local vascular function following acute exercise. Appl Physiol Nutr Metab. 2011;36(1):137-44.

20. Goto C, Higashi Y, Kimura M, et al. Effect of different intensities of exercise on endothelium dependent vasodilation in humans: role of endothelium dependent nitric oxide and oxidative stress. Circulation. 2003;108(5):530-5.

21. Green DJ, Bilsborough W, Naylor LH, et al. Comparison of forearm blood flow responses to incremental handgrip and cycle ergometer exercise: relative contribution of nitric oxide. J Physiol. 2005;562(Pt 2):617-28.

22. Green DJ, Hopman MTE, Padilla J, Laughlin MH, Thijssen DHJ. Vascular adaptation to exercise in humans: role of hemodynamic stimuli. Physiol Rev. 2017;97(2):495-528.

23. He $\mathrm{X}, \mathrm{Ku} \mathrm{DN}$. Pulsatile flow in the human left coronary artery bifurcation: average conditions. J Biomech Eng. 1996;118(1):74-78.

24. Johnson BD, Padilla J, Wallace JP. The exercise dose affects oxidative stress and brachial artery flow-mediated dilation in trained men. Eur J Appl Physiol. 2012;112(1):33-42.

25. Mills A, Rosenberg M, Stratton G, et al. The effect of exergaming on vascular function in children. J Pediatr. 2013;163(3):806-10.

26. Oertel G. Morphometric analysis of normal skeletal muscles in infancy, childhood and adolescence: an autopsy study. J Neurol Sci. 1988;88(1-3):303-13.

27. Ouriel K (2001) Peripheral arterial disease. Lancet 358, 1257-1264.

28. Padilla J, Simmons GH, Bender SB, Arce-Esquivel AA, Whyte JJ, Laughlin MH. Vascular effects of exercise: endothelial adaptations beyond active muscle beds. Physiology. 2011;26(3):132-45. 
29. Rasmussen AR, Wohlfahrt-Veje C, Tefre de Renzy-Martin K, et al. Validity of selfassessment of pubertal maturation. Pediatr. 2015;135(1)86-93.

30. Rubin DA, Castner DM, Pham H, Ng J, Adams E, Judelson DA. Hormonal and metabolic responses to a resistance exercise protocol in lean children, obese children and lean adults. Pediatr Exerc Sci. 2014;26(4):444-54.

31. Taddei S, Virdis A, Ghiadoni L, Magagna A, Salvetti A. Vitamin C improves endotheliumdependent vasodilation by restoring nitric oxide activity in essential hypertension. Circulation. 1998;97(22):2222-9.

32. Thijssen DH, Dawson EA, Black MA, Hopman MTE, Cable NT, Green DJ. Heterogeneity in conduit artery function in humans: impact of arterial size. Am J Physiol Heart Circ Physiol. 2008;295(5):H1927-34.

33. Thijssen DH, Bullens LM, van Bemmel MM, et al. Does arterial shear explain the magnitude of flow-mediated dilation: a comparison between young and older humans. Am J Physiol Heart Circ Physiol. 2009;296(1):H57-64.

34. Thijssen DH, Black MA, Pyke KE, et al. Assessment of flow-mediated dilation in humans: a methodological and physiological guideline. Am J Physiol Heart Circ Physiol. 2011;300(1):H2-12.

35. Timmons BW, Tarnopolsky MA, Bar-Or O. Immune responses to strenuous exercise and carbohydrate intake in boys and men. Pediatr Res. 2004;56(2):227-34.

36. Tinken TM, Thijssen DHJ, Hopkins N, Dawson EA, Cable NT, Green DJ. Shear stress mediates endothelial adaptations to exercise training in humans. Hypertension. 2010;55(2):312-8 
37. Tolfrey K, Armstrong N. Child-adult differences in whole blood lactate responses to incremental treadmill exercise. Br J Sports Med. 1995;29(3):196-9.

38. Vogel RA, Corretti MC, Plotnick GD. Effect of a single high-fat meal on endothelial function in healthy subjects. Am J Cardiol. 1997;79(3):350-4.

39. Wanne OPS, Haapoja E. Blood pressure during exercise in healthy children. Eur J Appl Physiol. 1988;58(1-2):62-7.

40. Watts K, Beye P, Siafarikas A, Davies EA, Jones TW, O’Driscoll G, Green DJ. Exercise training normalises vascular dysfunction and improves central adiposity in obese adolescents. J Am Coll Cardiol. 2004;43(10):1823-7.

41. Ziegler T, Bouzourene K, Harrison VJ, Brunner HR, Hayoz D. Influence of oscillatory and unidirectional flow environments on the expression of endothelin and nitric oxide synthase in cultured endothelial cells. Arterioscler Thromb Vasc Biol. 1998;18(5):686-92. 
Table 1. Physiological responses and perceived exertion during HIIE and MISS (values are mean $\pm \mathrm{SD}$ ).

\begin{tabular}{lcc}
\hline & HIIE (n=9) & MISS (n=9) \\
\hline Average watts (W) & $109 \pm 22^{*}$ & $53 \pm 11$ \\
RPE (1-10) & $7 \pm 2 *$ & $4 \pm 2$ \\
Mean HR (\% HR max $)$ & $82 \pm 4^{*}$ & $69 \pm 4$ \\
HR post (beats.min $\left.{ }^{-1}\right)$ & $99 \pm 10^{*}$ & $88 \pm 9$ \\
HR post60 (beats.min $\left.{ }^{-1}\right)$ & $79 \pm 7$ & $75 \pm 7$ \\
\end{tabular}

HR, heart rate, RPE, ratings of perceived exertion

*Significant difference between HIIE and MISS, $P<0.05$ 
Table 2. Baseline and reactive hyperemic responses of the superficial femoral artery before (Pre), after (Post) and 60-minutes after (Post60) high intensity interval exercise (HIIE) and moderate intensity steady state exercise (MISS). Data are presented as mean \pm SD

\begin{tabular}{|c|c|c|c|c|c|c|c|c|c|}
\hline & & \multirow{2}{*}{\multicolumn{3}{|c|}{$\begin{array}{l}\text { HIIE } \\
(n=9)\end{array}$}} & \multirow{2}{*}{\multicolumn{3}{|c|}{$\begin{array}{l}\text { MISS } \\
(n=9)\end{array}$}} & \multirow{3}{*}{$\begin{array}{c}\text { Time } \\
(P \text { value })\end{array}$} & \multirow{3}{*}{$\begin{array}{l}\text { Time } \mathrm{x} \\
\text { Intensity } \\
(P \text { value })\end{array}$} \\
\hline & & & & & & & & & \\
\hline & & Pre & Post & Post60 & Pre & Post & Post60 & & \\
\hline & Diameter $(\mathrm{mm})$ & $4.25 \pm 0.42$ & $4.76 \pm 0.58 \dagger$ & $4.25 \pm 0.36$ & $4.29 \pm 0.49$ & $4.61 \pm 0.67 \dagger$ & $4.28 \pm 0.53$ & $<0.001$ & 0.27 \\
\hline \multirow[t]{4}{*}{ Baseline } & Flow $\left(\mathrm{ml} . \mathrm{min}^{-1}\right)$ & $138.8 \pm 34.5$ & $512.3 \pm$ & $221.6 \pm$ & $157.4 \pm 46.5$ & $384.2 \pm$ & $218.7 \pm$ & $<0.001$ & $<0.05$ \\
\hline & & & $163.9 \dagger *$ & $51.2 \dagger$ & & $139.9 \dagger$ & $88.0 \dagger$ & & \\
\hline & OSI (AU) & $0.12 \pm 0.07$ & $0.00 \pm 0.00 \dagger$ & $0.03 \pm 0.02 \dagger$ & $0.10 \pm 0.06$ & $0.01 \pm 0.01 \dagger$ & $0.03 \pm 0.03 \dagger$ & $<0.001$ & 0.50 \\
\hline & Peak diameter $(\mathrm{mm})$ & $4.60 \pm 0.45$ & $5.03 \pm 0.51 \dagger^{*}$ & $4.62 \pm 0.37$ & $4.65 \pm 0.53$ & $4.84 \pm 0.57 \dagger$ & $4.67 \pm 0.57$ & $<0.001$ & $<0.05$ \\
\hline Reactive & FMD $(\%)$ & $8.42 \pm 2.10$ & $5.90 \pm 3.25 \dagger$ & $8.82 \pm 1.87$ & $8.24 \pm 2.34$ & $5.43 \pm 4.78 \dagger$ & $8.85 \pm 2.46$ & $<0.01$ & 0.96 \\
\hline \multirow[t]{4}{*}{ hyperemia } & Corrected FMD (\%) & $7.68 \pm 2.74$ & $7.57 \pm 2.74$ & $8.00 \pm 2.74$ & $7.68 \pm 2.74$ & $6.29 \pm 2.74$ & $8.22 \pm 2.74$ & 0.34 & 0.50 \\
\hline & $\mathrm{SR}_{\mathrm{AUC}}\left(\mathrm{ml} \cdot \mathrm{min}^{-1}\right)$ & $32511 \pm$ & $51524 \pm$ & $39494 \pm$ & $33206 \pm$ & $53880 \pm$ & $38291 \pm$ & $<0.001$ & 0.72 \\
\hline & & 11027 & $15486 \dagger$ & $9541 \dagger$ & 7609 & $9901 \dagger$ & $9964 \dagger$ & & \\
\hline & Flow AUC $\left(\mathrm{ml} \cdot \mathrm{min}^{-1}\right)$ & $525 \pm 127$ & $1075 \pm 224 \dagger$ & $648 \pm 130 \dagger$ & $561 \pm 159$ & $1045 \pm 353 \dagger$ & $632 \pm 131 \dagger$ & $<0.001$ & 0.73 \\
\hline
\end{tabular}


FMD, flow-mediated dilation; SR, shear rate; AUC, area under curve; AU, arbitrary units.

*Significant difference between HIIE and MISS, $P<0.05$. $\uparrow$ Significant difference compared to Pre, $P<0.05$. 


\section{Figure Captions \& Legends}

Figure 1. Schematic depicting the study design with the various measurement points for blood pressure (BP), heart rate (HR), perceived exertion (PE) and superficial femoral artery flowmediated dilation (SFA FMD).

Figure 2. Change in baseline diameter (panels a and b) and FMD (panels c and d) and from preto post-exercise and from pre- to 60-minutes post-exercise following HIIE (grey bars) and MISS (white bars). Data are means and SD. Dots represent individual data points.

Figure 3. Mean shear rate (panel a), antegrade (panel b), and retrograde (panel c) shear before (Pre), after (Post) and 60-minutes after (Post60) high-intensity interval exercise (HIIE, grey bars) and sustained moderate intensity exercise (MISS, white bars). Data are means and SD. *Significant difference between HIIE and MISS, $P<0.05$. $†$ Significant difference from preexercise, $P<0.001$. $\$$ Significant difference between Post and Post60, $P<0.001$. 